\title{
iPill: An iOS Mobile Application for Automatic Pill Identification Based on Image Processing Technique and Eigenface Algorithm
}

\author{
Ornicha Choungaramvong, Supangkana Tongsuk, Surasak Srilamlert and Supphachai Thaicharoen
}

\begin{abstract}
Consumable medical drugs are currently available in a variety of forms such as liquid, capsule, and tablet. For the tablet/pill form, many of them are manufactured in very similar appearances, which could lead to incorrect medical consumption, particularly for elderly and visually-impaired people. For the past several years, a number of software applications have been developed by both academic and business sectors to facilitate pill identification. These tools are ranged from Web-based applications, desktop applications, and to mobile applications. The key approach used by most of these tools is using image processing techniques to extract pill characteristics from a pill's image, converting these features into some numerical forms such as a vector model, and comparing the features with those stored in the database using some similarity or distance measures to determine a matched pill. Rather than using direct pixel-based characteristics and vector model for pill comparisons, an investigation of bringing in a face recognition algorithm to assist pill identification is presented in this paper. The proposed method exploits a commonly-known face recognition algorithm, known as Eigenface algorithm, to mathematically derive features from pills' images. These acquired features are used for determining the pill's shape, which are then combined with pill's color as criteria for the overall process of pill identification. In addition, the proposed technique is implemented as an iOS mobile application, named iPill, for an automatic pill identification. A preliminary result using 320 pill images from 20 types of pills with 9 different shapes and 10 dissimilar colors shows that iPill mobile application gives a satisfactory performance on the accuracy for automatic pill identifications.
\end{abstract}

Keywords-Eigenface algorithm, image processing, mobile application, pill identification.

\section{INTRODUCTION}

$C_{\text {i }}$ ONSUMABLE medical drugs are presently manufactured in a variety of forms such as liquid, capsule, and tablet. For the tablet or pill form, many of them are produced in similar appearances, which could lead to incorrect consumption, particularly for elderly and visually-impaired people.

According to our study, software applications developed for facilitating pill identification could be categorized into three main categories based on their application platforms: (i) mobile application $[3,4,7,8]$, (ii) desktop application $[1,2$,

Ornicha Choungaramvong, Supangkana Tongsuk, Surasak Srilamlert and Supphachai Thaicharoen are with Department of Computer Science, Faculty of Science, Srinakharinwirot University, 114 Sukhumvit 23, North Klongtoey Subdistrict, Wattana District, Bangkok 10110, Thailand.
11, 13, 16, 18], and (iii) Web application [5, 6, 9, 14, 17]. The key principle that most of these applications used for distinguishing pills is the use of physical characteristics of the pills such as color, shape, dimension, texture, score, and/or imprint, provided by the user or extracted by some image processing techniques. Subsequently, these physical appearances may be combined with other techniques such as constructing feature vector spaces for similarity or distance measures to find the matched pills in databases.

For instances, in the mobile application platform, Cunha and his research team proposed a mobile application for pill identification based on shape, dimension, and color of the pills [4]. In their method, shape is determined using shape-matching function, dimension is calculated using the minimum bounding box of the pill, and color is identified using pixel-by-pixel comparisons. According to their evaluation results, the pillmatching process works well only when there is a small set of pills stored in the database. Another research study in pill identification from images is proposed by Caban and team, which could be categorized as a desktop application. In their study, the modified shape distribution model was used for constructing a combined feature vector of colour, shape, and imprint. Then, the combined feature vector model is used to classify and identify the prescription drugs [1]. Their experimental results show a good accuracy for determining the top 5 of the matched pills. Nevertheless, the technique has not been implemented for practical uses or automatic identification. For the Web application platform, Pill Identification tool from WebMD is another example of pill identification [17]. To identify a pill with this online tool, the user must provide the imprint on the pill and then choose pill's color and shape as criteria for finding matched pills in an online database. Finally, in addition to these types of tools studied by academic sectors, several pill identification software tools are developed by business sectors for commercial purposes such as MedSnap-ID [12]. In MedSnapID, computer vision is utilized for extracting visual characteristics from the pill's image, and then use the extracted features to search for a matched pill in an online database. However, this tool might be costly for general uses by typical users.

According to our study, many pill identification tools uses pixel-based characteristics directly extracted from the pill images, constructs feature vectors from these characteristics, and then computes similarity or closest distance in identify the 
matched pills. This pixel-based characteristics could be sensitive to noises and feature vectors could be erroneous from offsets of values from vector computations. Hence, this paper presents another strategy as an iOS mobile application for aiding in automatic pill identification, named iPill. Instead of using direct pixel-based characteristics and feature vectors, iPill exploits a well-known object recognition algorithm, called Eigenface algorithm, to mathematically derive features of a particular pill's characteristic, its shape, from pills' images. Then, to identify the matched pill, it uses these derived features for determining pill's shape, and then combines it with another physical characteristic, pill's color, for identifying the desired pill. The overall approach of iPill is based on works by Cunha on mobile application platform [4], Kim and Chun on using basic features, color and shape [6], and Turk and Pentland on using Eigenface algorithm for object recognition [15].

The content of this paper is organized as follows. Technical background is described in Section 2 that covers a brief overview of image processing concepts and Eigenface algorithm. The developmental methodology is explained in Section 3 which comprises data and tools, application development methodology, and evaluation approaches. The experimental results are reported in Section 4 that shows the overall structure of iPill mobile application and its technical performance for automatic pill identification. Finally, the conclusion is drawn in Section 5 which gives an overview of the application and some discussion of key issues.

\section{TECHNICAL BACKGROUND}

\section{A. Image Processing}

Image processing is a computing process that transforms a physical image into a digital form as a two-dimensional array for further processing such as enhancing the image or extracting some characteristics [10]. In a typical image processing procedure, the input is an image or video frame and the output is the transformed digital image or data regarding the characteristics of the input image. For the approach used in this paper, image processing techniques are applied for extracting color and shape from pills' images.

Colors of the images that are relevant to the proposed method could be divided into two color systems, RGB (Red, Green, Blue) and HSV (Hue, Saturation, Value). RGB is a color system in which each color results from a combination of three key colors, red, green, and blue in different proportions. Although RGB color system is simple and appropriate to be used for representing or displaying images in electronic systems, it is not suitable for image processing because it is difficult to know the actual proportions of the three key colors that are made up of a particular color.

Generally, the colors of the images taken by using digital cameras are RGB colors. Accordingly, they should be converted into another color system for further processing. One of the appropriate color system in this case is HSV color system. In HSV color system, each color in the HSV system is represented by three properties, hue, saturation, and value. Hue is corresponding to the type of the color, saturation to the intensity of the color, and value to the brightness of the color, respectively. The HSV color system can be described as a cylinder of colors as follows.

- The perimeter of the circle of the cylinder represents the color range or hue which is divided into three main colors: red, green, and blue. The range of 0-120 degrees are corresponding to red color, the range of 121-240 degrees to the green color, and the range of 241-360 degrees to the blue color, subsequently.

- The distance from the central core of the cylinder to its parameter represents the intensity or saturation of the color. The greater the distance from the central core, the higher the intensity of the color is.

- The values along the vertical axis of the cylinder represents the brightness of the color. The value of the brightness starts from high at the top of the cylinder to low at the bottom of the cylinder.

In addition to the color systems, other image processing approaches employed in this paper are: converting a color image into a grayscale image, reducing an image's noises using Gaussian Blur, transforming a grayscale image to a binary image using Ostu's threshold method, detecting object boundary using canny edge detection, and finding an object using findContour() method. These functions and approaches are fortunately readily available for use in OpenCV library.

\section{B. Eigenface Algorithm}

Eigenface is a machine-learning algorithm for recognizing human faces. Its underlying principle is based on information theory where information is encoded as numerical data. Eigenface algorithm utilizes Principle Component Analysis to find a set of Orthonormal Eigenvectors, called Eigenfaces. These Eigenfaces represent a standard set of characteristic features of all faces, known as Eigenspace, in the training set. Typically, Eigenfaces are chosen from the top M Eigenvalues resulted from Singular Value Decomposition, which generally is less than the number of human faces in the training set [15]. The main idea of Eigenface algorithm is that rather than recognizing human faces based on typically physical characteristics such as eyes, noses, ears, mouths, or others, it characterizes the variations between faces.

An overall procedure for finding Eigenfaces and recognizing human faces in Eigenface algorithm can be described as follows.

\section{Learning mode}

Give an initial set of images of human faces with the same $\mathrm{N} \times \mathrm{N}$ dimensions as a training set.

Compute a set of $\mathrm{M}$ Eigenfaces from the training images. These M Eigenfaces are selected from the top M Eigenvalues of the output from Singular Value Decomposition. They are considered as a standard set of characteristic features, which represents the Eigenspace.

Transform each original image in the training set into Eigenspace by projecting each original image with each Eigenface in the Eigenspace. The result is a collection of weights representing the original image in the Eigenspace. 


\section{Recognizing mode}

Transform an unknown original image into Eigenspace by projecting the image with each Eigenface in the Eigenspace. The result is a collection of weights of the unknown image in Eigenspace.

Compute the distance between weights of the unknown image in the Eigenspace and weights of each training image in the Eigenspace

The identified image is the one which has the smallest difference or whose difference is less than the threshold.

\section{METHODS}

The developmental methodology for developing iPill application is divided into three parts: (i) data and tools, (ii) application development, and (iii) evaluation methods.

\section{A. Data and Tools}

A total of 320 pill images are used for training and testing. The images are taken from 20 different types of medicine in tablet form by using an iPhone camera, 16 images for each pill. These 20 pills are composed of 9 different shapes and 10 different colors. The number of pills used in experiments for training and testing based on colors and shapes are shown in Tables I and II, respectively.

TABLE I: Number of pills' images for each pill's color used in experiments

\begin{tabular}{ll} 
Color & Number of Images \\
\hline Orange-Brown & 32 \\
Red-Orange & 32 \\
White & 80 \\
Magenta-Pink & 16 \\
Yellow & 32 \\
Orange-Yellow & 32 \\
Pink & 48 \\
Cyan-Blue & 16 \\
Yellow-Green & 16 \\
Cyan & 16 \\
\hline \hline
\end{tabular}

TABLE II: Number of pills' images for each pill's shape used in experiments

\begin{tabular}{ll}
\hline Shape & Number of Images \\
\hline 6Sides & 32 \\
Barrel & 16 \\
Clover & 32 \\
Double-Circle & 16 \\
Oblong & 64 \\
Oval & 48 \\
Round & 64 \\
Square & 16 \\
Triangle & 32 \\
\hline \hline
\end{tabular}

\section{B. Application Development}

iPill is developed on Macbook Air with OSX El Capitan operating systems and XCode IDE 7.1. It is written using Objective-C and Swift programming languages. OpenCV 3.0 library $(\mathrm{C}++$ version) is exploited for image processing and machine learning tasks, and Gimp is used for designing the application user interface. The overall application development procedure can be divided into the following five steps.
- Image acquisition -- In order to reduce any variance due to environmental factors such as light, shadow, and distance from the pill to the phone camera, a card box is used for photographing the pills. Natural light is controlled and the distance is fixed as much as possible.

- Color extraction -- The feature extraction from pills' images is divided into two steps. In the first step, the location of the pill in the image is searched. The original colored image in RGB system is converted into Grayscale. After that, to reduce any noises, Gaussian Blur is used. Next, Canny edge detection is employed to detect the boundary of the pill. Finally, the location of the pill is found by using findContour method. In the second step, the coordinate of the pill in the Grayscale image is used to find the pill's color in the original colored image which is already converted from RGB to HSV colored system.

- Shape learning -- Learning pills' shapes is carried out by converting a set of pills' colored images in RGB system into Grayscale system. Then, the Grayscale images are fed into Eigenface algorithm for training and building a classification model. Eigenface algorithm is readily available in OpenCV library.

- Pill identification -- To identify pills, two characteristics are used: shape and color. The shape of the pill is used as the first discriminative criterion according to the classification model built from Eigenface algorithm, and the color is used as the second condition.

- Output demonstration -- The results obtained from the pill identification process is divided into two types, matched and unmatched. If the testing pill is the pill recognized by the classification model, its information is displayed on the smartphone screen such as its image, indication, and dosage. These information is previously stored in the local database on the smartphone.

\section{Evaluation Method}

The performance of iPill application for pill identification is based on the accuracy measure as shown in Eq. 1.

$$
\text { Accuracy }=\frac{\text { Total number of correct identifications }}{\text { Total number of identifications }}
$$

\section{EXPERIMENTAL RESULTS}

The experimental results are divided into two parts: (i) the mobile application for pill identification and (ii) the performance evaluation results.

\section{A. A Mobile Application for Pill Identification}

The mobile application for pill identification, iPill, consists of 11 user-interface pages as shown in Fig. 1, which is divided into 4 main parts according to its functionality.

- The starting page and main page -- This part consists of splash screen and main page where the user can switch to one of the remaining parts, personal information, pill information, and pill identification and result.

- The user personal information -- In this part, the user can fill in his/her information such as name, address, and emergency contact information. 


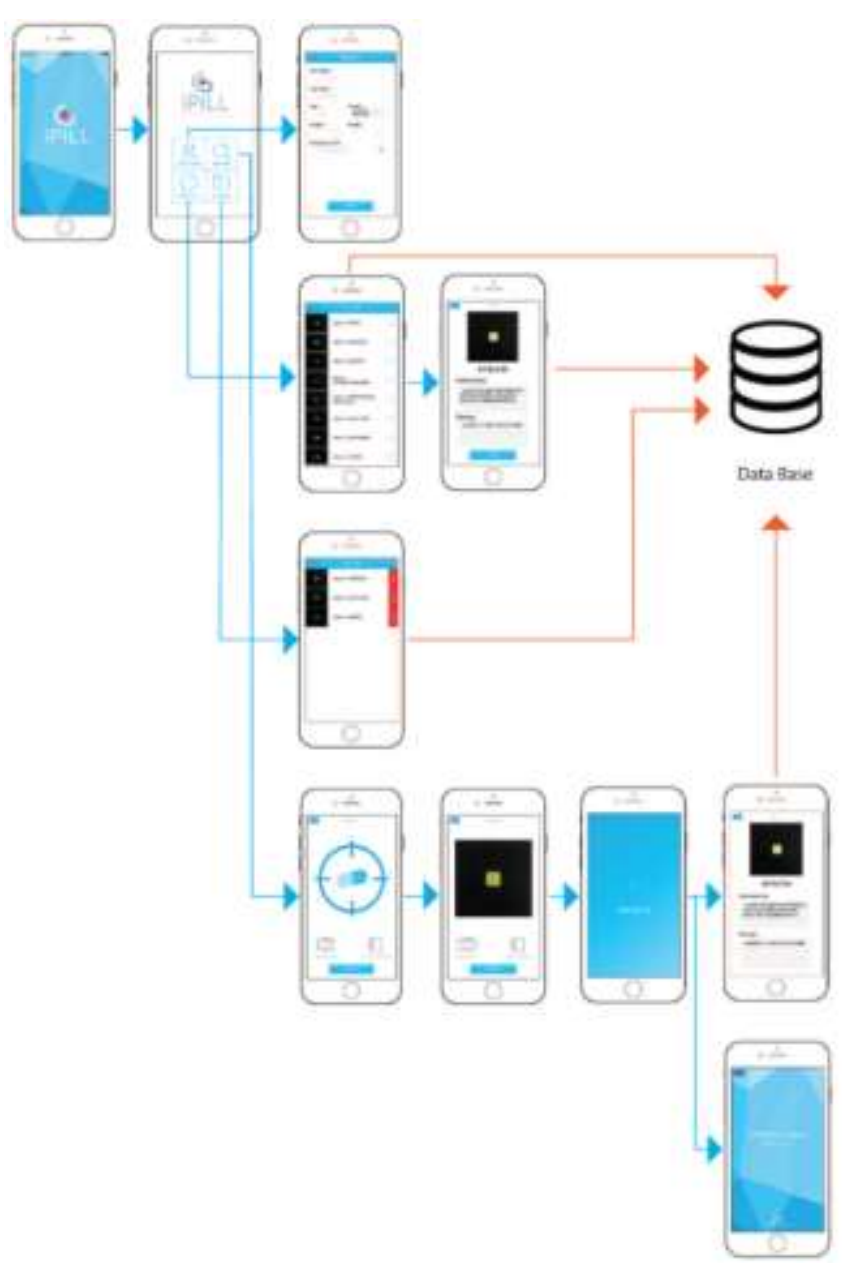

Fig. 1: The overall architecture of iPill application.

- Pill information -- In this part, the user can retrieve a list of drugs stored in the local database including the details of each medicine.

- Pill identification and result -- This part allows the user to take a picture of the pill to be used for identification, show the taken pill image, identify pill and display the results.

\section{B. Performance Evaluation Results}

The performance evaluation results on the accuracy of pill identification is summarized in Table III. The results show that iPill application gives the overall average accuracy at $76.88 \%$. Out of this, its highest accuracy is $100 \%$ for 3 types of pills and over $80 \%$ for 10 types of pills. There are only 3 types of pills that iPill yields the accuracy below $60 \%$.

TABLE III: Evaluation results on pill identifications using shapes and colors

\begin{tabular}{lllll}
\hline \hline Pill Name & Color & Shape & $\begin{array}{l}\text { Testing } \\
\text { Quantity }\end{array}$ & $\begin{array}{l}\text { Correct } \\
\text { Identification }\end{array}$ \\
\hline COVIR & Orange-Brown & 6Sides & 16 & $8(50.00 \%)$ \\
VIROGON & Red-Orange & 6Sides & 16 & $12(75.00 \%)$ \\
ANAPRIL & White & Barrel & 16 & $10(62.50 \%)$ \\
CYPROHEPTADINE & Magenta-Pink & Clover & 16 & $14(87.50 \%)$ \\
$\begin{array}{l}\text { METOPROLOL 100 } \\
\text { STADA }\end{array}$ & White & Clover & 16 & $14(87.50 \%)$ \\
McXY Cold & Yellow & DoubleCircle & 16 & $9(56.25 \%)$ \\
COPROFEN & Yellow & Oblong & 16 & $5(31.25 \%)$ \\
$\begin{array}{l}\text { DURAN } \\
\text { GLUCOSAMINE/ }\end{array}$ & White & Oblong & 16 & $12(75.00 \%)$ \\
CHONDROITIN & Orange-Yellow & Oblong & 16 & $15(93.75 \%)$
\end{tabular}

\begin{tabular}{lllll} 
DECAVITAMIN & Pink & Oblong & 16 & $11(68.75 \%)$ \\
IBUTAMOL & Orange & Oval & 16 & $16(100.00 \%)$ \\
CODIPHEN & Cyan-Blue & Oval & 16 & $16(100.00 \%)$ \\
MEFAMED & Orange-Yellow & Oval & 16 & $15(93.75 \%)$ \\
DECOLGEN & Yellow-Green & Round & 16 & $13(81.25 \%)$ \\
IBUMAN & Pink & Round & 16 & $12(75.00 \%)$ \\
SPAMUS & Cyan & Round & 16 & $14(87.50 \%)$ \\
CEMOL & White & Round & 16 & $10(62.50 \%)$ \\
MYBACIN & Yellow-Green & Square & 16 & $16(100.00 \%)$ \\
MAGESTO-F & White & Triangle & 16 & $13(81.25 \%)$ \\
SAMBEE & Pink-Red & Triangle & 16 & $11(68.75 \%)$ \\
\hline \hline
\end{tabular}

\section{CONCLUSION}

iPill is an iOS mobile application for facilitating automatic pill identification based on shapes and colors of the pills. Its underlying principle is on the basis of image processing techniques for extracting colors and shapes from the pills and Eigenface algorithm for learning and recognizing the shapes of the pills. The fundamental notion of this approach is to examine the use of face recognition algorithms in the problem domain of pill identification. From the experimental results, iPill application can identify pills with the highest accuracy of $100 \%$ for three types of pills and higher than $80 \%$ for 10 types of pills from the total of 20 types of pills. For the pills that iPill does not give high accuracy, it might be the result from the inconsistency in the photographing step which causes pills' images to be blurred. Another source of low performance might come from different parameter configurations of the camera for different shots such as different focus ranges or unequal light intensity.

These satisfactory results may suggest that face recognition algorithms could be applied to assist and supplement pill identification processes in addition to image processing techniques. Another interesting and useful application of face recognition algorithms in pill identification domain is to apply the algorithms for directly recognizing pills that have characters, scores, or imprints, which is analogously similar to human faces.

\section{REFERENCES}

[1] J. J. Caban, A. Rosebrock, and T. S. Yoo, "Automatic Identification of Prescription Drugs Using Shape Distribution Models," in 2012 19th IEEE International Conference on Image Processing. IEEE, 2012, pp. $1005-1008$ https://doi.org/10.1109/icip.2012.6467032

[2] J. Camargo, P. Esseiva, F. González, J. Wist, and L. Patiny, "Monitoring of illicit pill distribution networks using an image collection exploration framework," Forensic Science International, vol. 223, no. 1-3, pp. 298305, Nov 2012.

https://doi.org/10.1016/j.forsciint.2012.10.004

[3] C. Crema, A. Depari, A. Flammini, M. Lavarini, E. Sisinni, and A. Vezzoli, "A smartphone-enhanced pill-dispenser providing patient identification and in-take recognition," in 2015 IEEE International Symposium on Medical Measurements and Applications (MeMeA). IEEE, May 2015, pp. 484-489. https://doi.org/10.1109/memea.2015.7145252

[4] A. Cunha, T. Adão, and P. Trigueiros, "HelpmePills: a mobile pill recognition tool for elderly persons," Procedia Technology, vol. 16, pp. 1523-1532, 2014. https://doi.org/10.1016/j.protcy.2014.10.174

[5] Drugs, "Pill Finder [Web application]. Available at https://www.drugs.com/pill_identification.html," 2016. 
[6] GoodRX, "Pill Identifier [Web application]. Available at http://www.goodrx.com/pill-identifier," 2016.

[7] A. Hartl, C. Arth, and D. Schmalstieg, "Computer-Vision based Pharmaceutical Pill Recognition on Mobile Phones," Central European Seminar on Computer Graphics (CESCG), 2010.

[8] A. Hartl, C. Arth, and D. Schmalstieg, "Instant Medical Pill Recognition on Mobile Phones," International Conference on Computer Vision (ICCV), pp. 188-195, 2011. https://doi.org/10.2316/p.2011.740-017

[9] Healthline, "Pill Identifier [Web application]. Available at http://www.healthline.com/pill-identifier," 2016.

[10] Kavita, R. Saroha, R. Bala, and S. Siwach, "Review paper on Overview of Image Processing and Image Segmentation," International Journal of Research in Computer Applications and Robotics, vol. 1, no. 7, pp. 113, 2013.

[11] D. Kim and J. Chun, "Drug Image Retrieval by Shape and Color Similarity of the Medication," in 2011 First ACIS/JNU International Conference on Computers, Networks, Systems and Industrial Engineering (CNSI). IEEE, May 2011, pp. 387-390. https://doi.org/10.1109/CNSI.2011.40

[12] MedSnap, "Medsnap-ID [Mobile application]. Available at https://medsnap.com/," 2016.

[13] T. Murai, M. Morimoto, and K. Fuji, "A Visual Inspection System for Prescription Drugs," 2012 Fifth International Conference on Emerging Trends in Engineering and Technology, pp. 13-18, 2012. https://doi.org/10.1109/ICETET.2012.29

[14] NLM, "Pillbox [Web application]. Available at https://pillbox.nlm.nih.gov/pillimage/search.php," 2016.

[15] M. Turk and A. Pentland, "Face recognition using eigenfaces," Proceedings. 1991 IEEE Computer Society Conference on Computer Vision and Pattern Recognition, pp. 586-591, 1991. https://doi.org/10.1109/cvpr.1991.139758

[16] C. Wang, S. Kamata, and L. Ma, "A Fast Multi-View Based Specular Removal Approach for Pill Extraction," 2013 IEEE International Conference on Image Processing, pp. 4126-4130, 2013. https://doi.org/10.1109/ICIP.2013.6738850

[17] WebMD, "Pill Identification Tool [Web application]. Available at http://www.webmd.com/pill-identification/," 2016.

[18] J. Yu, Z. Chen, and S. Kamata, "Pill Recognition Using Imprint Information by Two-Step Sampling Distance Sets," 2014 22nd International Conference on Pattern Recognition (ICPR), pp. 31563161, 2014.

https://doi.org/10.1109/icpr.2014.544 